\title{
Simple generation of two-color FEL pulses using a sextupole magnet
}

\author{
Philipp Dijkstal, Sven Reiche, and Eduard Prat \\ Paul Scherrer Institut, Forschungsstrasse 111, Villigen, Switzerland
}

\begin{abstract}
We present a new and simple method to generate two-color free-electron laser (FEL) pulses. In our scheme an electron bunch is quadratically tilted in the transverse direction and exhibits an energy chirp. The head and tail of the bunch are aligned to the undulator beamline and will lase, while the middle part of the bunch undergoes orbit oscillations and thus is unable to amplify the FEL radiation. Since the beam is energy chirped, the central wavelengths of the SASE amplification at the two aligned parts of the bunch are different. The beam tilt is introduced by a variation of sextupole strength in a bunch compressor section. We present simulation results of this new method for the hard X-ray FEL beamline Aramis at SwissFEL. This includes the general performance and tunability of the scheme, the requirements on the energy chirp and tilt generation, and adverse effects associated with the beam tilt.
\end{abstract}

Keywords: FEL, free-electron laser, two-color pulses, hard X-ray, transverse beam tilt, energy chirp, sextupole magnet, SwissFEL

\section{INTRODUCTION}

\subsection{Free-electron lasers}

X-ray free-electron lasers (FEL) ${ }^{1}$ offer unique photon properties to scientists from many fields such as biology or material sciences. The perspective of wavelengths down to the Angstrom level in combination with pulse durations down to the fs level and high peak powers on the order of tens of GW have led to the design and construction of hard X-ray facilities such as LCLS (USA), ${ }^{2}$ SACLA (Japan), ${ }^{3}$ and more recently PAL-XFEL (Korea) ${ }^{4}$ European XFEL (Germany) ${ }^{5}$ and SwissFEL (Switzerland). ${ }^{6}$

An FEL is based on the interaction of an electron bunch with an electromagnetic field inside an undulator. Undulators consist of short dipolar magnets of alternating polarity. The electron bunches follow a curved path and first emit incoherent synchrotron radiation. This radiation is then amplified and filtered by the FEL process, resulting in a coherent pulse. The pulse energy exponentially grows along the undulator beamline up to the saturation level. Since the initial signal arises from spontaneous emission, this mode of operation is called Self-Amplified Spontaneous Emission ${ }^{7,8}$ (SASE). The resonance condition relates the central FEL radiation wavelength $\lambda$ with the undulator period $\lambda_{u}$, the electron beam Lorentz factor $\gamma$ and the undulator field strength $K$ for planar undulators:

$$
\lambda=\frac{\lambda_{u}}{2 \gamma^{2}}\left(1+\frac{K^{2}}{2}\right)
$$

Various other conditions need to be fulfilled for the SASE process to occur. A high charge density in the sixdimensional phase space is required, which translates to a high current, a small energy spread, low transverse emittances and small beam sizes. The bunch also needs to be well matched and have a well-aligned orbit to maintain a suitable transverse overlap with the photon field.

\footnotetext{
E-mail: philipp.dijkstal@psi.ch
} 


\subsection{Two-color FEL pulses}

There is a scientific interest in two-color FEL pulses. Two short laser pulses, simultaneous or with an adjustable time delay, are required for experiments in research fields such as material science or molecular physics. Among the use cases are pump-probe ${ }^{9}$ or coherent stimulated X-ray Raman spectroscopy (CXRS) ${ }^{10,11}$ experiments. We give a short overview of other schemes that can provide two-color FEL pulses before introducing our method.

It follows from the resonance condition (Eq. 1) how two-color pulses can be achieved with a SASE FEL. Since all undulators of a beamline typically exhibit the same period $\lambda_{u}$, there are two fundamental ways. First, two undulator sections with different field strengths can be used with a beam of equal energy. Second, two electron bunches, or two parts of the same bunch, at different energies can be used with the undulator section tuned at the same strength.

The first group of methods has the advantage of good tunability: the range of the undulator field strength can be large when variable-gap devices are installed, and the time separation can be widely tuned with a delaying chicane between the undulators. A drawback is that only half of the undulator length is available for each pulse. ${ }^{12,13}$ Another downside is that the beam quality in the second undulator part is already degraded from the SASE process in the first undulator part. As a result, the peak power of the second FEL pulse is lower unless the first pulse is not driven into saturation, limiting in this case the FEL power of the first stage. With a fresh-slice scheme it is possible to overcome this disadvantage. Here, the bunch is divided in two parts, each of which is only set up to lase in one of the undulator sections. Therefore, the beam quality of the second part is preserved while it passes the first undulator group and the FEL power of the first section can reach saturation. The suppression of the SASE process can be achieved with either a slice mismatch ${ }^{14}$ (see Eq. 5) or a linear beam tilt. ${ }^{15,16}$

The second type of methods has the advantage that it is in principle more compact or affordable than the group of methods mentioned above, since the same undulator modules are used for both pulses. It is not as easily tunable, however. One possibility is to use two consecutive bunches at different beam energies. ${ }^{17,18} \mathrm{An}$ alternative is an energy-chirped single pulse, meaning that the particle energy is correlated with the longitudinal position. The FEL process needs to be suppressed for the middle part of the bunch, while allowing the head and the tail to generate two-color radiation. To suppress partially the radiation, it has been proposed to use a slotted foil to manipulate the slice emittance. ${ }^{19}$ Alternatively, the longitudinal bunch emittance ${ }^{20}$ or current profile $^{21}$ can be tailored to obtain the desired temporal FEL pulse profile.

In this article we present a new scheme that is also based on an energy-chirped single bunch. The FEL radiation from the middle part of the bunch is suppressed with a quadratic beam tilt. The merits of this scheme are the simplicity and the relatively minor hardware requirements since only one sextupole magnet is used. We explain the method in detail in the following section, before showing results of a start-to-end simulation study for the Aramis beamline at SwissFEL. We also address the adverse side effects of our method of beam tilt generation. Furthermore, the tunability of the scheme is analyzed.

\section{WORKING PRINCIPLE}

\subsection{Explanation of the scheme}

The underlying idea of our scheme is that the disruption of overlap between a longitudinal slice of the electron bunch and the radiation field reduces the slice pulse power significantly with respect to a well aligned slice. Undulator sections are typically operated as FODO beamlines, i.e., an alternating sequence of quadrupoles and undulators. For a quadratically tilted beam, it is possible to align the head and the tail of the bunch, while the middle part is displaced and receives kicks from the quadrupoles in the undulator beamline. The overlap between the particles of the central slices and the radiation field is thereby diminished. The exponential power increase that is characteristic to the SASE process is thereby stopped. The two aligned parts are undisturbed and emit radiation that reaches the saturation level. Two pulses separate in time are generated from the head and the tail of the bunch. If the beam is energy-chirped, these pulses are also of different colors.

We suggest a simple method to impose a quadratic tilt that only requires one sextupole in a bunch compressor section. When comparing with the other methods described in Sec. 1.2, the necessary equipment is rather 

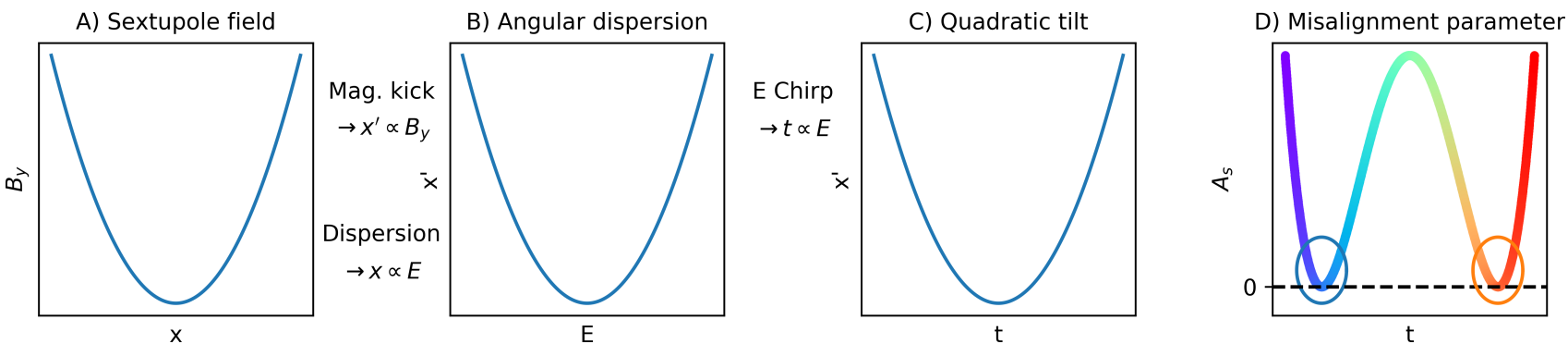

Figure 1. The method of generating a quadratic tilt with an energy-chirped bunch in a dispersive section with a sextupole is explained schematically. A combination of linear dispersion and a sextupolar magnetic field results in a second-order angular dispersion in the horizontal plane after the sextupole (A and B). Due to the linear energy chirp, quadratic dispersion translates to a quadratic beam tilt (C). The misalignment parameter (Eq. 2) is visualized after a shift of the whole beam in order to obtain two aligned slices (D).

minimal. In short, three steps are necessary: the quadratic tilt needs to be generated, two parts of the bunch have to be aligned, and an energy chirp must be imposed.

Bunch compressors are ideal locations to impose a quadratic tilt. Bunch compression ${ }^{22}$ at FELs is typically achieved with a combination of an RF section and a magnetic chicane. The beam is accelerated off-crest in the RF stations, which generates an energy chirp with the tail of the bunch exhibiting a higher energy. Within magnetic chicanes, the beam exhibits linear beam dispersion, which is a correlation between horizontal position $x$ and energy $E$. As a result of transverse dispersion, the path length is energy-dependent and the bunch is shortened. The principle of generating the quadratic tilt with a sextupole inside a chicane is explained in Fig. 1. The first graph shows the sextupole field strength $B_{y}$ that depends quadratically on the particle position $x$. The magnetic field translates to a change in $x^{\prime}$ immediately after the sextupole (second graph). The variable $x$ is exchanged with $E$ since the beam exhibits linear dispersion, and $x^{\prime}$ is exchanged with $B_{y}$ since the kick is proportional to the field strength. With an energy chirp, a dependence of $x^{\prime}$ on the particle energy translates to a dependence on time (third graph).

Downstream of the magnet, the sextupole kick manifests as a combination of positional and angular offsets to the centroid position of a slice that depends on its longitudinal position. We define the slice misalignment parameter $A_{s}$ as a measure of the amplitude of the displacement of a whole slice, independent of the betatron ${ }^{23}$ phase:

$$
A_{s}(t)=\frac{\beta\left\langle x_{s}^{\prime}(t)\right\rangle^{2}+2 \alpha\left\langle x_{s}(t)\right\rangle\left\langle x_{s}^{\prime}(t)\right\rangle+\gamma\left\langle x_{s}(t)\right\rangle^{2}}{\epsilon_{x, c}}
$$

where $\alpha, \beta$ and $\gamma$ are the Twiss parameters of the beam and $x_{s}(t), x_{s}^{\prime}(t)$ the coordinates of particles within a slice. Since the bunch is sliced according to the temporal particle positions $t$, the particle coordinates have a time dependence. The enumerator of Eq. 2 is the Courant-Snyder invariant of motion, ${ }^{23}$ a parameter that is here calculated for slice centroids. We normalize by the emittance of the central slice $\left(\epsilon_{x, c}\right)$, located in the middle of the bunch. The slice emittance is a measure of the volume in the two-dimensional horizontal phase space that is occupied by the particles belonging to this slice. We choose the slice emittance of the central part of the beam instead of the projected beam emittance since it is not affected by the imposed beam tilt. The displacement parameter $A_{s}$ is dimensionless and invariant under linear optics transformations, as it is the case for undulator beam optics. It is zero for an aligned slice.

The central part of the bunch passes the field-free region of the sextupole and continues on the nominal orbit after the chicane, i.e., only this part is aligned. To obtain two aligned parts, the whole beam needs to be shifted. With downstream corrector magnets it is possible to center an arbitrary slice in both angle and offset before the beam enters the undulator section. Mathematically this corresponds to adding constants to the $x, x^{\prime}$ coordinates of all particles so that $\left\langle x_{s}(t)\right\rangle$ and $\left\langle x_{s}^{\prime}(t)\right\rangle$ are zero for a specific slice. If the beam tilt is symmetric in both angle and offset, the centering of the bunch tail occurs when the head is centered. At the same time, the central slices are misaligned. This is visualized in the right graph in Fig. 1. The change in color symbolizes the different FEL 
wavelengths that can be generated by each slice due to the energy chirp. The aligned orbit is depicted with a dashed line. Only slices sufficiently close to this orbit (inside the circles) can generate FEL radiation.

The final energy chirp can be achieved in several ways. It is possible to use the RF modules in off-crest acceleration mode. This is straight-forward but not very efficient; as a further drawback the maximum achievable beam energy is reduced. Other more suitable methods use passive wakefield dechirpers ${ }^{24-26}$ or overcompression in combination with wakefields. ${ }^{27}$

\subsection{Detrimental effects}

There are detrimental effects related to the use of sextupoles. The magnetic field can be expressed as:

$$
\begin{gathered}
B_{x}(x, y)=g_{2} x y \quad ; \quad B_{y}(x, y)=\frac{1}{2} g_{2}\left(x^{2}+y^{2}\right) \\
g_{2}=\frac{\partial B_{y}(x)^{2}}{\partial^{2} x} \quad ; \quad k_{2}=\frac{q}{p} g_{2},
\end{gathered}
$$

with $k_{2}$ being the normalized sextupole strength, $g_{2}$ the second-order field gradient, $q$ the particle charge and $p$ the particle momentum.

As mentioned above, the bunch compression scheme relies on a path length difference for particles with different energies. The use of a sextupole changes this path length and leads to an alteration of the current profile, ${ }^{28}$ which later also affects the FEL performance. This can be counteracted by imposing also a quadratic chirp before the chicane, for example with an X-band cavity.

The magnetic field couples the $x$ and $y$ planes for particles with $y \neq 0$. Coupling is generally undesired in most particle accelerators, including FEL machines. The effect can be reduced by having a small vertical beam size and a well-aligned sextupole, in which case the induced coupling is small.

Furthermore, sextupoles generate an emittance increase in the horizontal plane that scales with the third power of the beam size and linearly with the normalized sextupole strength. ${ }^{29}$ It is therefore beneficial to have small beam sizes in both planes at the sextupole location.

Sextupoles also cause chromaticity, which is the derivative of the phase advance with respect to the particle energy. The induced chromaticity has linear dependences on the sextupole strength and on the $\beta$ and dispersion functions of the beam while it passes the sextupole magnet. If the chromatic effects caused by the sextupole are found to be intolerable, with four sextupoles it is in principle possible to control the chromaticity in both transverse planes while maintaining the same quadratic tilt in both angle and offset. ${ }^{30}$ This optimization is out of scope of this presentation since most detrimental effects are tolerable for the simulations shown later (Sec. 3).

Due to the linear energy chirp, chromaticity translates to a slice mismatch that depends on the longitudinal position within the bunch. The slice mismatch parameter $M_{s}$ is defined in the following. It is a function of the Twiss parameters of a reference $\left(\beta_{r}, \alpha_{r}, \gamma_{r}\right)$ and those of the slice $\left(\beta_{s}, \alpha_{s}, \gamma_{s}\right)$, the latter again with an implicit dependence on $t$.

$$
M_{s}(t)=\frac{1}{2}\left(\gamma_{s}(t) \beta_{r}-2 \alpha_{s}(t) \alpha_{r}+\beta_{s}(t) \gamma_{r}\right)
$$

$M_{s}$ has a value of 1 for a perfectly matched slice. The quadratic tilt generated with a sextupole depends linearly on the energy chirp, the dispersion function and the sextupole strength. Since all detrimental effects scale with the sextupole strength, it is beneficial to use a high chirp and dispersion so that the required strength is reduced. Moreover, the beam size in both planes should be minimized.

\subsection{Tunability}

The scheme provides two pulses that are separate in time and wavelength and are characterized by their individual pulse durations. In principle, each of these parameters is tunable.

The wavelength separation is a consequence of the energy difference of the two aligned parts. As an example, from the resonance condition (Eq. 1) an energy separation of $1 \%$ would correspond to a wavelength separation of $2 \%$. 
There is a threshold of the misalignment parameter $A_{s}$ above which lasing is suppressed. This threshold is dependent on the FEL wavelength: a higher misalignment is required to prevent lasing at longer wavelengths. ${ }^{30-33}$ The individual pulse duration is determined by the range in time where $A_{s}$ remains below this threshold (see Fig. 1). One can expect short pulse durations when the slope of the beam tilt is large at the locations of the aligned slices. The slope is increased towards the outer parts of the bunch or when higher sextupole strengths are used.

The temporal separation of the two FEL pulses depends on the positions of the slices that are aligned. An obvious upper limit is the total pulse duration. On the other hand, when the aligned slices approach each other while the imposed quadratic beam tilt remains constant, the displacement parameter $A_{s}$ of the intermediate part decreases. In case $A_{s}$ of the central slice does not surpass the threshold of lasing suppression, a broadband FEL pulse is emitted instead of two distinct pulses. The lower limit of the temporal separation is therefore determined by the maximum quadratic tilt that can be generated.

The temporal separation and the individual pulse duration are coupled as both depend on the locations of the aligned slices. To maintain the same pulse duration when the temporal separation is increased, the sextupole strength needs to be lowered.

\section{SIMULATION STUDIES}

\subsection{Simulations setup for SwissFEL}

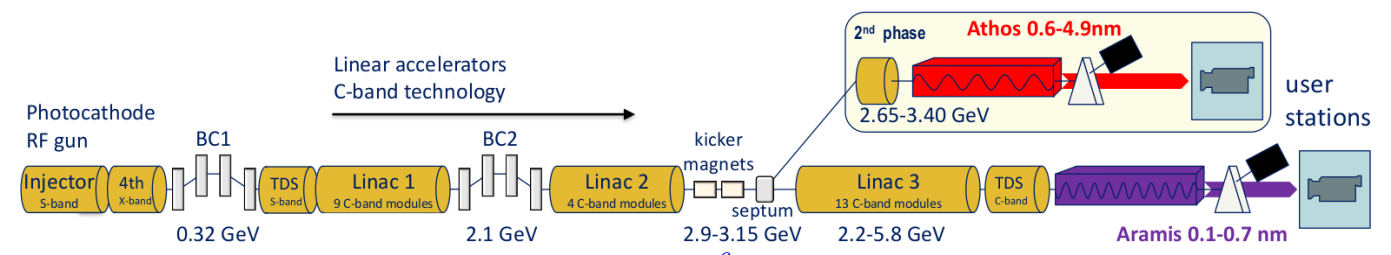

Figure 2. A schematic overview of the SwissFEL accelerator. ${ }^{6}$ Most relevant for this article are bunch compressor BC1 and the undulator section in the Aramis Beamline.

SwissFEL is designed to provide bright X-ray laser pulses for a wide range of user experiments. Figure 2 gives a schematic overview of the machine. The hard X-ray beamline Aramis has been in operation since the end of 2017. Athos is the soft X-ray beamline currently still in its installation phase, with some parts already being commissioned. The electron source for SwissFEL is an RF photocathode. The particles are then accelerated to an energy of up to $5.8 \mathrm{GeV}$ (Aramis) or $3.4 \mathrm{GeV}$ (Athos). Two bunch compressors (BC1 and BC2) are installed to shorten the bunch length. The FEL radiation wavelength is adjustable from 1 to $7 \AA .^{6}$

The software Elegant ${ }^{34}$ is used to simulate the electron beam from the SwissFEL injector until the end of Linac 3. Coherent synchrotron radiation (CSR) effects ${ }^{35}$ inside chicanes and wakefields inside RF cavities are considered in the simulation. The starting particle distribution is generated with the ASTRA software ${ }^{36}$ and consists of $6 \times 10^{5}$ macroparticles. The final particle distribution resulting from the Elegant simulations is matched and centered using linear transformations of the particle coordinates. An energy chirp of $0.5 \%$ (full-width) is added in the same way. For the future realization of the scheme, several methods could be employed to generate the final energy chirp, as mentioned in Sec. 2.1. The modified particle distribution is then passed as an input to Genesis $1.3,{ }^{37}$ which is used to simulate the FEL process in the undulator section. Important simulation and beam parameters are given in Table 1 . The average of three simulations with random seed numbers are used whenever results from Genesis simulations are shown in the following pages. The study is set up for a wavelength of about $1 \AA$.

Two sextupoles are installed in each bunch compressor section, one after the first dipole and one before the last dipole. At nominal beam energy (see Fig. 2), the maximum sextupole strengths $K_{2}$ are $50 \mathrm{~m}^{-2}$ in $\mathrm{BC} 1$ and $412 \mathrm{~m}^{-2}$ in BC2. Simulation studies were performed where all four sextupoles were varied. We find that the second sextupole in $\mathrm{BC} 1$ is most suitable for the two-color scheme, since the generated tilt is sufficient while the detrimental effects are tolerable. The sextupole strength in $\mathrm{BC} 1$ is lower compared to $\mathrm{BC} 2$, but this is compensated with a higher energy chirp and a higher beam dispersion compared to the second bunch 
Table 1. Beam parameters for simulations with Elegant and Genesis 1.3.

Bunch charge

Normalized initial slice emittance

$200 \mathrm{pC}$

$300 \mu \mathrm{m}$

Central beam energy before the undulator section

$5.8 \mathrm{GeV}$

Average projected $\beta$

Undulator $K$

$12.7 \mathrm{~m}\left(\beta_{x}\right), 9.7 \mathrm{~m}\left(\beta_{y}\right)$

Undulator period $\lambda_{u}$

Central FEL wavelength $\lambda_{c}$

1.2

$0.015 \mathrm{~m}$

$0.98 \AA$

compressor. Moreover, the horizontal $\beta$ function is smaller at the second sextupole in $\mathrm{BC} 1$ compared to the first. In the following, only simulation results where the second sextupole in $\mathrm{BC} 1$ is used are discussed.

\subsection{Demonstration of the scheme}
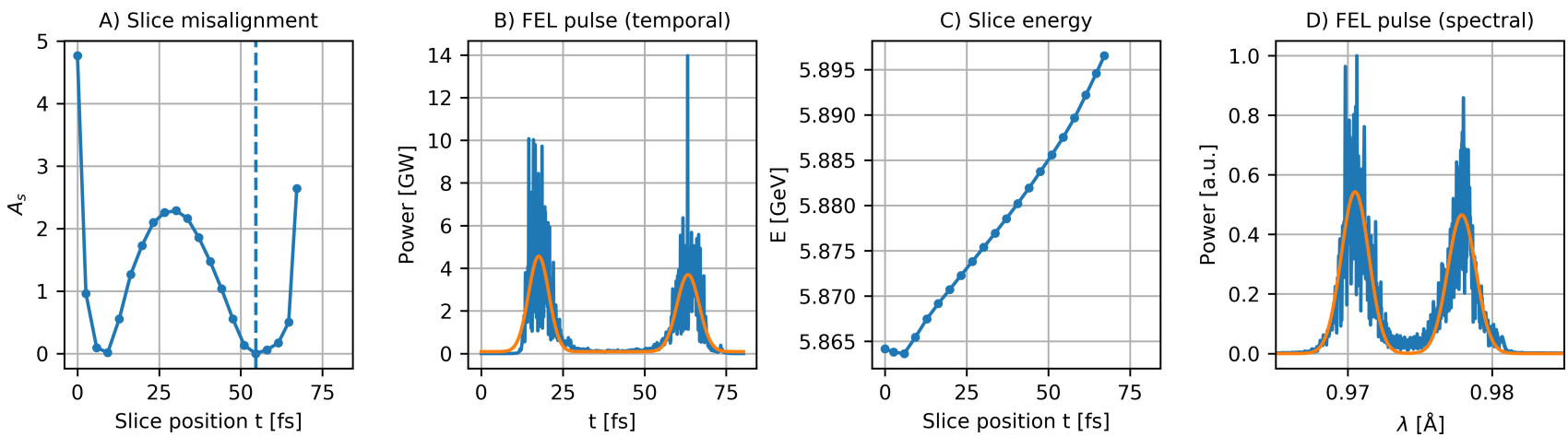

Figure 3. Results from start-to-end simulations where the tilt is generated with the first sextupole in BC1. Plot (A) shows the normalized slice invariant (see Eq. 2). The centered slice is indicated with a dashed line. In (B), the corresponding temporal FEL pulse shape is displayed. The energy chirp and the wavelength spectrum are shown to the right. The spikes in the spectrum and pulse power are superimposed with Gaussian fits for better visibility of the signal.

Figure 3 shows results from start-to-end simulations that confirm the general feasibility of the method. The sextupole strength of the second magnet in $\mathrm{BC} 1$ is set to $40 \mathrm{~m}^{-2}$. The projected particle distribution after Linac 3 is matched to periodic beam optics in the Aramis FODO beamline. The bunch is then divided in 21 slices based on the longitudinal particle position. One slice is centered by shifting the horizontal positions and angles of all particles, thereby setting its displacement parameter $A_{s}$ to exactly zero. Due to the quadratic order of the beam tilt, this results in an approximately similar displacement parameter of the opposite slice. The shape of the misalignment parameter $A_{s}$ corresponds to a temporal FEL pulse with two power spikes. The energy chirp is reflected in the wavelength spectrum, which also consists of two spikes. The power generated from the middle part of the bunch is significantly reduced. Two Gaussian distributions are fitted to the temporal FEL pulse data to obtain a measure of the pulse properties. The peak power of the Gaussian fits to the two pulses are 4.3 and $3.5 \mathrm{GW}$, the temporal separation of the peaks is $46 \mathrm{fs}$, the separation in wavelength is $0.77 \%$ and the individual pulse durations are 7.4 and $8.1 \mathrm{fs}$ (FWHM), respectively. For this beam energy and undulator settings, a misalignment parameter of about 1 is sufficient to prevent a slice from lasing.

\subsection{Analysis of detrimental effects}

We find that the adverse effects on the bunch are rather minimal in the case we studied in Sec. 3.2, where the sextupole strength is set to $40 \mathrm{~m}^{-2}$. This is however not the case when the higher sextupole strengths 100 and $150 \mathrm{~m}^{-2}$ are used. The detrimental effects on beam current, slice mismatch and slice emittance are shown in Fig. 4 for the sextupole strengths $0,40,100$ and $150 \mathrm{~m}^{-2}$. In general, the expected behavior described in Sec. 2.2 is observed. With higher sextupole strengths, charge is shifted from the tail to the head of the bunch and the resulting current profile becomes increasingly asymmetric. A larger mismatch is visible for the outmost slices in all cases. However, the beam current at these positions is very low, so these slices would not be able 

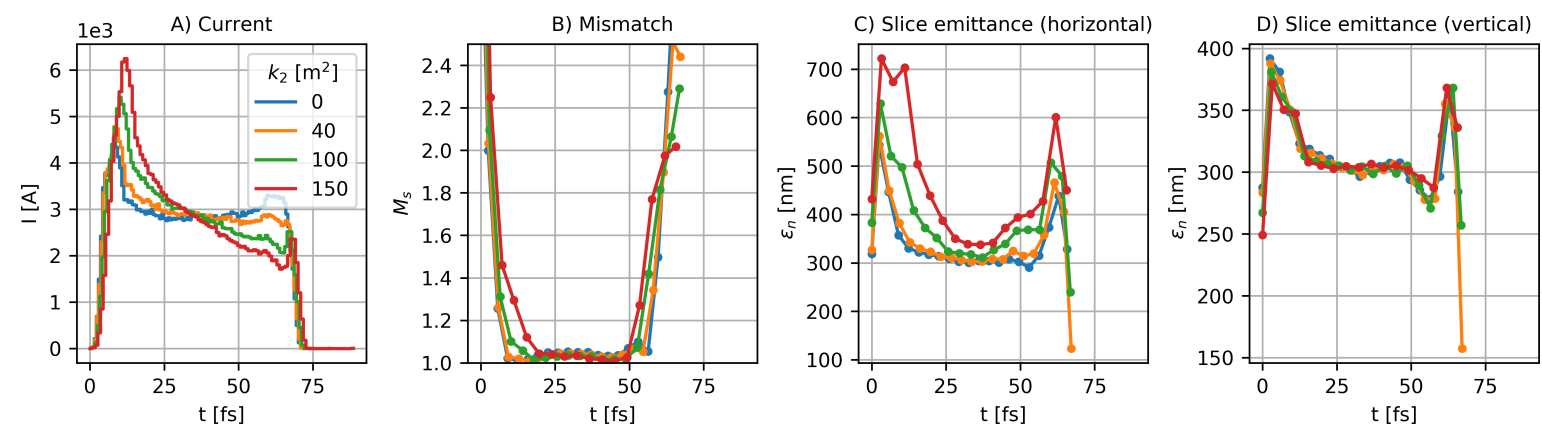

Figure 4. Beam current, slice mismatch (see Eq. 5) and transverse slice emittances for different sextupole strengths.

to generate radiation even without a mismatch. For a strength of $150 \mathrm{~m}^{-2}$, the increase in mismatch is however shifted towards the inner parts of the bunch. A significant emittance growth for most slices is also observed for the higher sextupole strengths, with the bunch head being most affected. The vertical emittance in contrast is barely changed, as expected. Of the observed effects, the horizontal slice emittance increase and the beam current alteration are the most relevant. The current imbalance is beneficial to the SASE process of the first pulse but detrimental to the second. From the emittance increase in the cases of $k_{2}=100 \mathrm{~m}^{-2}$ and $k_{2}=150 \mathrm{~m}^{-2}$ one can expect a highly negative impact on the performance.

\subsection{Discussion of tunability}

We study the tunability of the separation in time and the individual pulse duration by variation of the sextupole strength and slice alignment: the strengths $20,40,80$ and $150 \mathrm{~m}^{-2}$ are simulated, and in each case, four different slices are centered. Figure 5 shows the misalignment parameter together with the pulse power profile for all cases. Furthermore, some quantities derived from the pulse profile are shown. The individual pulse duration is calculated as the full width at half maximum (FWHM) of the Gaussian fits. The separation of the two pulses is defined as the difference of the two means of the fit. The contrast ratio is calculated as the average of the two Gaussian fit peaks divided by the average power of the central slice that is located in the middle of the two pulses.

In general, the expected behavior (see Sec. 2.3) is visible. Higher sextupole currents and centering of the outer slices both reduce the individual pulse duration while increasing the power contrast ratio. For low sextupole strengths $\left(20 \mathrm{~m}^{-2}\right)$, it becomes clear that the scheme breaks down when the centered slices are too close to the middle part of the bunch. The orbit displacement of the central slices is then not sufficient to suppress lasing, and all slices contribute to the FEL pulse. This is reflected in longer pulse durations and lower contrast ratios between the two pulses and the central part. Furthermore, the scheme does not work at high sextupole strengths $\left(150 \mathrm{~m}^{-2}\right)$, since the pulse power starts to decrease, especially for the second pulse, and there is no tunability. This is primarily a consequence of the altered beam current and the increased horizontal emittance, as discussed in Sec. 3.3.

In Sec. 2.3 we explained that the individual pulse duration and the pulse separation are coupled. To explore this behavior, simulations were performed for sextupole strengths in the range of 20 to $100 \mathrm{~m}^{-2}$ and different aligned slices. Only one random seed for the Genesis 1.3 software is used in contrast to the five used for the other results presented. For this exercise we require for a two-color pulse that both peaks have a maximum power (of the Gaussian fit) above $1 \mathrm{GW}$ each. In addition, the average of the two peaks is required to be at least 10 times larger than the power emitted by the central part. The temporal separation and the average pulse duration from all simulations that fulfill these criteria is plotted in graph (L) of Fig. 5. The temporal separation ranges between 30 and $50 \mathrm{fs}$, and the average single pulse duration ranges between 4 and $12 \mathrm{fs}$. However, we did not find a combination of sextupole strength and slice alignment for which a two-color pulse with a separation of less than $40 \mathrm{fs}$ and average individual pulse durations of less than 6 fs are obtained.

We conclude that a limited tunability of the time separation is available. If the centered slices are very close to each other, the intermediate parts still lase. The minimal pulse separation depends on the use case, i.e., the minimal contrast ratio that is acceptable for the experiment. 

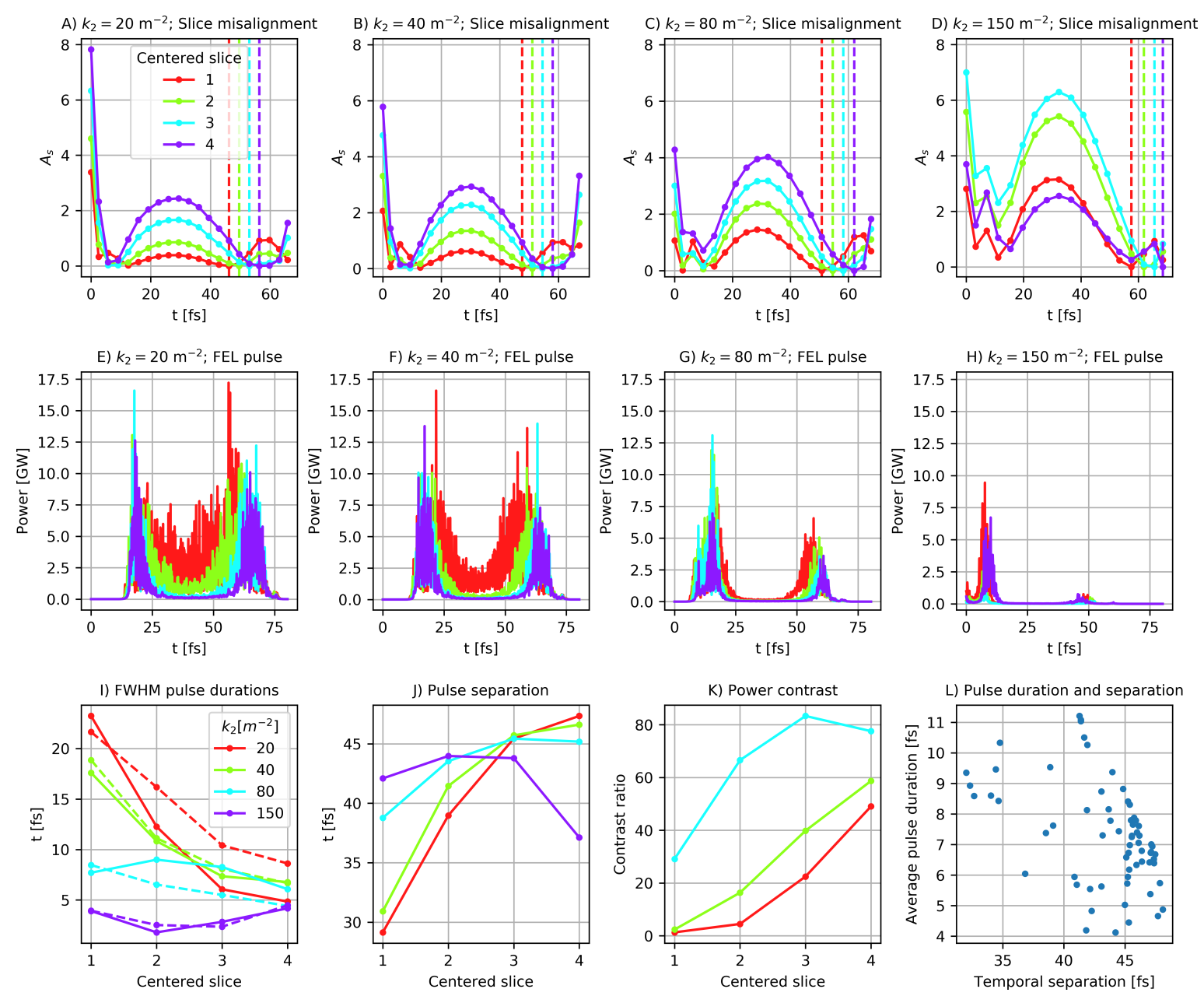

Figure 5. A-D: the misalignment parameter (Eq. 2) for different centered slices, indicated with dashed lines. Here, the invariant is 0 by definition. E-H: the resulting temporal shape of the FEL pulses. I: the FWHM of the Gaussian fits to the first (straight lines) and second (dashed lines) pulses for different sextupole strengths and centered slices. J: the separation of the means of the Gaussian fits. K: the average of the peaks of the two fits is divided by the pulse power generated by the middle slice. L: separation and average duration of the two pulses for a simulation study where $k_{2}$ and the aligned slice number are scanned.

The results for a $k_{2}$ of $150 \mathrm{~m}^{-2}$ show that it is possible to generate one single short pulse with a quadratic tilt when a high quadrupole strength is used. In this case, the detrimental effects of the second-order tilt generation on the beam quality work to the advantage of a short-pulse scheme, since one of the two pulses is suppressed. A short pulse may, however, also be achieved with a linear beam tilt imposed by a quadrupole. ${ }^{38}$

\section{CONCLUSIONS}

We have presented a scheme that can be used to obtain a two-color FEL pulse from a single bunch using an undulator section with a homogeneous undulator field strength. It has the advantages that it is simple and requires little special instrumentation, namely one sextupole at a location where the beam is energy-chirped and exhibits dispersion. In the case of SwissFEL, sextupoles are anyway part of the machine design. A similar method to generate two-color pulses relies on sending the beam through a slotted foil that increases the transverse beam emittance for the whole bunch except two parts. ${ }^{19}$ Especially for machines that operate at a high-repetition rate or can for other reasons only afford minimal bremsstrahlung generation, the sextupole-based scheme is attractive.

A start-to-end simulation study using one sextupole for the Aramis hard X-ray beamline was performed to show the feasibility of the method. In addition, the tunability of the scheme and the detrimental effects on the 
beam quality that are associated with the use of sextupoles were discussed. We find that the scheme is generally working and that the individual FEL pulse durations and the separation of the pulses in time and wavelength are tunable. The negative impact on the beam quality is tolerable in the case of the sextupole magnet we used for the study. However, it is also shown that above a certain strength the sextupole will greatly degrade the beam quality. This would render the two-color scheme unusable, but at the same time opens up the possibility to generate single short pulse.

We plan to demonstrate the scheme experimentally at SwissFEL and to show the measurement results in a separate publication.

\section{Acknowledgements}

We thank S. Bettoni for supply and support concerning the initial file distributions from ASTRA simulations. A. Malyzhenkov has been very helpful in the discussion of CSR effects within a magnetic chicane. Many thanks go to Thomas Schietinger for a careful proof-reading of the paper.

\section{REFERENCES}

[1] Pellegrini, C., Marinelli, A., and Reiche, S., "The physics of x-ray free-electron lasers," Rev. Mod. Phys. 88, 015006 (2016).

[2] Emma, P. et al., "First lasing and operation of an ångstrom-wavelength free-electron laser," Nature Photonics 4(9), 641-647 (2010).

[3] Ishikawa, T. et al., "A compact x-ray free-electron laser emitting in the sub-ångström region," Nature Photonics 6(8), 540 (2012).

[4] Kang, H.-S. et al., "Hard x-ray free-electron laser with femtosecond-scale timing jitter," Nature Photonics 11(11), 708 (2017).

[5] Weise, H., "Commissioning and first lasing of the European XFEL," in [Proceedings of FEL 2017, Santa $\mathrm{Fe}, \mathrm{NM}, \mathrm{USA}],(2017)$.

[6] Milne, C. et al., "SwissFEL: The Swiss X-ray Free Electron Laser," Applied Sciences 7(7) (2017).

[7] Kondratenko, A. and Saldin, E., "Generation of Coherent Radiation by a relativistic Electron Beam in an ondulator," Particle accelerators Print 10(3-4), 207-216 (1980).

[8] Bonifacio, R., Pellegrini, C., and Narducci, L., "Collective instabilities and high-gain regime in a free electron laser," Optics Communications 50(6), 373 - 378 (1984).

[9] Inoue, I. et al., "Observation of femtosecond X-ray interactions with matter using an X-ray-X-ray pump-probe scheme," Proceedings of the National Academy of Sciences 113(6) (2016).

[10] Schweigert, I. V. and Mukamel, S., "Probing valence electronic wave-packet dynamics by all x-ray stimulated Raman spectroscopy: A simulation study," Physical Review A 76(1) (2007).

[11] Harbola, U. and Mukamel, S., "Coherent stimulated x-ray Raman spectroscopy: Attosecond extension of resonant inelastic x-ray Raman scattering," Physical Review B 79(8) (2009).

[12] Jaroszynski, D. A., Prazeres, R., Glotin, F., and Ortega, J. M., "Two-color free-electron laser operation," Physical Review Letters 72(15), 2387-2390 (1994).

[13] Hara, T. et al., "Two-colour hard X-ray free-electron laser with wide tunability," Nature Communications 4 (2013).

[14] Qin, W., Ding, Y., Lutman, A. A., and Chao, Y.-C., "Matching-based fresh-slice method for generating two-color x-ray free-electron lasers," Physical Review Accelerators and Beams 20(9) (2017).

[15] Reiche, S. and Prat, E., "Two-color operation of a free-electron laser with a tilted beam," Journal of Synchrotron Radiation 23(4), 869-873 (2016).

[16] Lutman, A. A. et al., "Fresh-slice multicolour X-ray free-electron lasers," Nature Photonics 10(11), 745-750 (2016).

[17] Marinelli, A. et al., "High-intensity double-pulse X-ray free-electron laser," Nature communications 6, 6369 (2015). 
[18] Decker, F.-J., Bane, K., Colocho, W., Lutman, A., and Sheppard, J., "Recent Developments and Plans for Two Bunch Operation with up to $1 \mu$ s Separation at LCLS," in [Proceedings of FEL 2017, Santa Fe, NM, USA], (2017).

[19] Hernández, Á. S., Prat, E., and Reiche, S., "Generation of two-color x-ray free-electron-laser pulses from a beam with a large energy chirp and a slotted foil," Physical Review Accelerators and Beams 22(3) (2019).

[20] Marinelli, A. et al., "Optical Shaping of X-Ray Free-Electron Lasers," Phys. Rev. Lett. 116, 254801 (2016).

[21] Bettoni, S., Prat, E., and Reiche, S., "Two-color beam generation based on wakefield excitation," Physical Review Accelerators and Beams 19(5) (2016).

[22] Mitri, S. D., "Bunch-length Compressors," in [Proceedings of CAS-CERN Accelerator School], (2018).

[23] Courant, E. and Snyder, H., "Theory of the alternating-gradient synchrotron," Annals of Physics 3(1), 1-48 (1958).

[24] Craievich, P., "Passive longitudinal phase space linearizer," Physical Review Special Topics - Accelerators and Beams 13(3) (2010).

[25] Emma, P., Venturini, M., Bane, K., Stupakov, G., Kang, H.-S., Chae, M., Hong, J., Min, C.-K., Yang, H., Ha, T., Lee, W., Park, C., Park, S., and Ko, I., "Experimental Demonstration of Energy-Chirp Control in Relativistic Electron Bunches Using a Corrugated Pipe," Physical Review Letters 112(3) (2014).

[26] Antipov, S. et al., "Experimental Demonstration of Energy-Chirp Compensation by a Tunable DielectricBased Structure," Physical Review Letters 112(11) (2014).

[27] Hernández, Á. S., Prat, E., Bettoni, S., Beutner, B., and Reiche, S., "Generation of large-bandwidth x-ray free-electron-laser pulses," Physical Review Accelerators and Beams 19(9) (2016).

[28] England, R. J., Rosenzweig, J. B., Andonian, G., Musumeci, P., Travish, G., and Yoder, R., "Sextupole correction of the longitudinal transport of relativistic beams in dispersionless translating sections," Physical Review Special Topics - Accelerators and Beams 8(1) (2005).

[29] Dowell, D., "Correcting Emittance Growth Due to Stray Sextupole Fields," Tech. Rep. SLAC-R-1091, Standford Linear Accelerator Center (2018).

[30] Guetg, M. W., Beutner, B., Prat, E., and Reiche, S., "Optimization of free electron laser performance by dispersion-based beam-tilt correction," Phys. Rev. ST Accel. Beams 18, 030701 (2015).

[31] Tanaka, T., Kitamura, H., and Shintake, T., "Consideration on the BPM alignment tolerance in X-ray FELs," Nuclear Instruments and Methods in Physics Research Section A: Accelerators, Spectrometers, Detectors and Associated Equipment 528(1-2), 172-178 (2004).

[32] Reiche, S., "A method for measuring the FEL radiation power along an undulator," Nuclear Instruments and Methods in Physics Research Section A: Accelerators, Spectrometers, Detectors and Associated Equipment 445(1-3), 139-142 (2000).

[33] MacArthur, J. P., Lutman, A. A., Krzywinski, J., and Huang, Z., "Microbunch Rotation and Coherent Undulator Radiation from a Kicked Electron Beam," Physical Review X 8(4) (2018).

[34] Borland, M., "elegant: A Flexible SDDS-Compliant Code for Accelerator Simulation," Tech. Rep. LS-287, Argonne National Laboratory (2000).

[35] Bane, K. L. F. et al., "Measurements and modeling of coherent synchrotron radiation and its impact on the Linac Coherent Light Source electron beam," Physical Review Special Topics - Accelerators and Beams 12(3) (2009).

[36] Flötmann, K., ASTRA A Space Charge Tracking Algorithm. DESY, Hamburg (2017).

[37] Reiche, S., "GENESIS 1.3: a fully 3D time-dependent FEL simulation code," Nucl. Instr. Meth. A 429(1-3), 243-248 (1999).

[38] Guetg, M. W., Lutman, A. A., Ding, Y., Maxwell, T. J., and Huang, Z., "Dispersion-based fresh-slice scheme for free-electron lasers," Phys. Rev. Lett. 120, 264802 (2018). 